\title{
Contribution of organic cation transporter 2 (OCT2) to cisplatin- induced nephrotoxicity
}

\author{
Kelly K. Filipski ${ }^{1,2}$, Ron H. Mathijssen ${ }^{3}$, Torben S. Mikkelsen ${ }^{1,4}$, Alfred H. Schinke ${ }^{5}$, and \\ Alex Sparreboom $1,2,3$
}

${ }^{1}$ Department of Pharmaceutical Sciences, St. Jude Children's Research Hospital, Memphis, TN ${ }^{2}$ University of Tennessee Health Science Center, Memphis, TN ${ }^{3}$ Department of Medical Oncology, Erasmus MC - Daniel den Hoed Cancer Center, Rotterdam, the Netherlands ${ }^{4}$ Department of Pediatric Oncology, Skejby Hospital, Aarhus University Hospital, Denmark ${ }^{5}$ Division of Molecular Biology, the Netherlands Cancer Institute, Amsterdam, the Netherlands

\begin{abstract}
Cisplatin is one of the most widely used anticancer agents for the treatment of solid tumors. The clinical use of cisplatin is associated with dose-limiting nephrotoxicity, which occurs in one-third of patients despite intensive prophylactic measures. Organic cation transporter 2 (OCT2) has been implicated in the cellular uptake of cisplatin, but its role in cisplatin-induced nephrotoxicity remains unknown. In mice, deletion of Oct1 and Oct2 resulted in significantly impaired urinary excretion of cisplatin without an apparent influence on plasma levels. Furthermore, the Oct1/Oct2-deficient mice were protected from severe cisplatin-induced renal tubular damage. Subsequently, we found that a non-synonymous single-nucleotide polymorphism in the OCT2 gene SLC22A2 (rs316019) was associated with reduced cisplatin-induced nephrotoxicity in patients. Collectively, these results indicate the critical importance of OCT2 in the renal handling and subsequent renal toxicity of cisplatin, and provide a rationale for the development of new targeted approaches to mitigate this debilitating side effect.
\end{abstract}

\section{Keywords}

Cancer Chemotherapeutics; Drug-Transporter Interactions

\section{INTRODUCTION}

Cis-diamminedichloroplatinum (cisplatin) is among the most widely used cytotoxic anticancer agents and has a broad spectrum of activity against various diseases, including lung, head and neck, bladder, germ cell, ovarian, endometrial, and cervical cancers (1). In the conventional 3or 4-weekly treatment regimens, dose-limiting side effects associated with cisplatin-based

Correspondence to Alex Sparreboom, St. Jude Children's Research Hospital, 262 Danny Thomas Place, Memphis, TN 38105, CCC, Room I5308. Phone: (901) 595-5346; Fax: (901) 595-3125; (e-mail: alex.sparreboom@ @stjude.org). AUTHOR CONTRIBUTIONS

K.K.F., A.H.S. and A.S. conceived and designed the experiments; K.K.F., R.H.M. and T.S.M. performed the experiments; K.K.F. and A.S. analyzed the data and wrote the manuscript; R.H.M. and A.H.S. contributed to writing and editing the manuscript; A.S. supervised the project.

CONFLICT OF INTEREST

Oct1/2 knockout mice are made commercially available through the company Taconic. The research group of A.H.S. benefits from a fraction of the revenue generated. The other authors declared no conflict of interests. 
chemotherapy regimens include renal tubular dysfunction (nephrotoxicity), peripheral neuropathy, and hearing loss (ototoxicity), whereas hematological toxicity becomes dose limiting in the more dose-dense, regimens in which the drug is administered on a weekly basis (2).

Severe and irreversible damage to the kidney remains the most important complication of cisplatin treatment as it may limit further treatment or even threaten life. This side effect primarily affects the $\mathrm{S} 3$ segment of the renal proximal tubules and occurs in one-third of patients despite intensive prophylactic measures (3). Furthermore, about $20 \%$ of all acute renal failure cases among hospitalized patients are due to cisplatin-containing chemotherapy (4). Despite having been the focus of intense investigation for many decades, the exact pathogenesis of cisplatin-related nephrotoxicity, in which quiescent proximal tubular cells are selectively damaged, remains unclear (5). Recent studies have suggested that inflammation, oxidative stress and apoptosis probably explain part of the tissue injury, although the initiating event leading to tubular damage and mechanistic details of the drug's renal handling are still poorly understood (6).

We and others have recently reported that the organic cation transporter 2 (OCT2), encoded by the $S L C 22 A 2$ gene, can actively transport cisplatin in vitro (7-10). This transporter is predominantly expressed in human kidney at the basolateral membrane of renal proximal tubules, and is involved in the secretion of various cationic substances from the circulation into tubular cells. All experiments to date, however, have assessed a putative role of OCT2 in cisplatin-related nephrotoxicity in cultured cells. The continual identification of substrates for renal transporters using heterologous in vitro expression systems provides valuable information for predicting drug-drug and drug-protein interactions (11). However, the wellrecognized limitation of these preliminary determinations is that they do not indicate the true relevance of a transporter in handling a substrate in the context of whole-body disposition, renal and extrarenal transport, and glomerular filtration. Indeed, to attribute an abnormality in normal physiology to transporter perturbation, the relevance of the transporter to the disposition of a drug must first be determined in vivo. The aim of the current investigation was to compare the pharmacokinetics, urinary excretion, and extent of nephrotoxicity in mice lacking the ortholog transporters Oct1 [Oct1(-I-) mice], Oct2 [Oct2(-I-) mice], or both Oct1 and Oct2 $[$ Oct 1/2(-I-) mice] after treatment with cisplatin. In addition, we evaluated the association of genetic variation in the $S L C 22 A 2$ gene with nephrotoxicity seen in patients treated with cisplatin.

\section{RESULTS}

\section{SLC22A2 Expression in Human Normal and Tumor Tissue}

To provide further insight into the tissue-specific expression profile of the OCT2 gene $S L C 22 A 2$, real-time PCR analysis was performed on a panel of normal human tissues. As expected, the tissue with the highest expression level was the kidney, whereas low expression levels were noted in a variety of other tissues, including the intracranial artery (Figure 1A). $S L C 22 A 2$ expression was further evaluated in a select series of human tumors, and we found a low level of expression in a number of kidney tumor types. Interestingly, the expression of $S L C 22 A 2$ was absent in breast, colon, liver, lung, ovarian, prostate, and thyroid tumors, particularly when compared to the baseline expression in the normal human kidney (Figure 1B). In line with a previous observation (12), very low to absent expression levels of $S L C 22 A 2$ were found in most cell lines of the NCI 60 human tumor panel, with the exception of a single ovarian cancer cell line, namely SKOV-3 (data not shown). 


\section{Urinary Cisplatin Excretion in Oct1- and Oct2- Deficient Mice}

We next assessed the urinary excretion profile of cisplatin in mice following a single dose of $10 \mathrm{mg} / \mathrm{kg}$. We found that the cumulative percentage of the administered dose was very similar in both Oct1(-/-) mice (Supp Figure 1A) and Oct2(-l-) mice (Supp Figure 1B) compared with age-matched wildtype mice. Consistent with previous findings (13), the majority of the cisplatin dose was excreted in urine within the first 24 hours after drug administration. The lack of a significant impact of the individual Oct1 and Oct2 deficiencies on the elimination of cisplatin is in line with earlier observations made for the cationic substance tetraethylammonium (TEA) in the same animal models $(14,15)$, and suggests that in the mouse cisplatin is likely recognized as a substrate by both Oct1 and Oct2. Although mice express substantial levels of both Oct1 and Oct2 in the kidney, in humans there is strong agreement that OCT2 dominates renal organic cation transport, whereas OCT1 dominates hepatic organic cation transport (16). Therefore, we used Oct1/2(-/-) mice in all subsequent experiments as the most appropriate animal model.

\section{Cisplatin Pharmacokinetics in Oct1/2(-/-) Double Knockout Mice}

At 24 hours after cisplatin administration, a cumulative urinary excretion amounting to $45 \%$ of the administered dose was observed in Oct $1 / 2(-/-)$ mice compared to $91 \%$ in wildtype animals $(P=0.0008)$, and only $56 \%$ of the dose was recovered in Oct $1 / 2(-/-)$ mice $(P=0.0016)$ (Figure 2A) within the sample collection period. The rate of the cumulative urinary excretion of cisplatin was also markedly reduced in the Oct $1 / 2(-/-)$ mice, confirming a direct role of organic cation transporters in the renal handling of cisplatin. Microarray analysis of kidney samples revealed that the Slc22a8 gene, encoding the organic anion transporter 3 (OAT3), was specifically upregulated (up to 11 -fold for the 2 probe sets) in the Oct1/2(-/-) mice (Figure 2B). This transporter is also localized on the basolateral membrane of renal proximal tubule cells and has been identified as an important contributor to xenobiotic and endogenous organic anion secretion (17). To rule out a role of OAT3 in the renal tubular transport of cisplatin, in vitro transport of cisplatin was assessed in mammalian cells engineered to overexpress human OAT3 or murine Oat3. These studies indicated, however, that cisplatin is not a substrate of either transporter $(P=0.093$ and $P=0.14$, respectively) (Figure 2C), and it was thus concluded that this factor was not confounding the results of the murine pharmacokinetic studies.

The reduced renal excretion of cisplatin in the Oct1/2(-/-) mice was not accompanied by measurable changes of cisplatin concentrations in plasma (Figure 3A). The observed peak concentration of cisplatin was slightly increased in Oct1/2(-I-) mice compared with wildtype mice (26.7 $\pm 9.73 \mu \mathrm{g} / \mathrm{mL}$ versus $36.4 \pm 8.47 \mu \mathrm{g} / \mathrm{mL}$, respectively; $P=0.20)$, although the total area under curve was very similar between groups $(50.4 \mu \mathrm{g} . \mathrm{h} / \mathrm{mL}$ versus $54.9 \mu \mathrm{g} . \mathrm{h} / \mathrm{mL}$, respectively). These values are comparable with the average area under the curve of cisplatin in 270 cancer patients receiving cisplatin at a dose of $100 \mathrm{mg} / \mathrm{m}^{2}(54.9 \pm 12.4 \mu \mathrm{g} . \mathrm{h} / \mathrm{mL})(18)$. The fraction unbound cisplatin was also not significantly different between plasma samples obtained from Oct1/2(-l-) mice and wildtype mice (10.3\% versus $11.1 \% ; P=0.46)$ (Figure $3 \mathrm{~B})$, suggesting that possible differences in the extent of drug binding to serum proteins did not contribute to the impaired urinary excretion.

\section{Histopathology and Serum Chemistry Analysis}

To evaluate the pharmacodynamic implications of the Oct1/2-genotype dependent urinary excretion pattern of cisplatin, various additional analyses were performed. As predicted based on the excretion data, we found that severe, acute renal tubular necrosis was observed in kidneys of all wildtype mice $(n=8)$ but in none of the Oct1/2(-l-) mice $(n=11)$ (Figure 4$)$. The lesions observed in cisplatin-treated wildtype mice were characterized by dilated tubules filled with necrotic tubular epithelial cells, cellular debris and proteinaceous casts, whereas the glomeruli, which do not express Oct1 or Oct2, were histologically normal. Evidence of toxicity to 18 other 
tissues in the Oct1/2(-I-) mice, including liver, bone marrow, and intestine, was absent (Supp Figure 2), indicating that the impaired renal excretion in this strain does not cause exacerbated cisplatin-related side effects elsewhere.

Consistent with the histopathological changes observed in the cisplatin-treated wildtype mice, various physiologic hallmarks of cisplatin-related nephrotoxicity in humans, such as changes in serum alkaline phosphatase, blood urea nitrogen, glucose, and serum creatinine, were specifically altered in wildtype mice undergoing cisplatin treatment (Table 1).

\section{SLC22A2 Genotype-Toxicity Associations}

Consistent with the view that cisplatin-related nephrotoxicity may be linked to how the kidney transports cisplatin is the high cisplatin content of human proximal tubules (19) and the correlation of nephrotoxicity with concentrations of cisplatin in autopsy kidney cortex samples (20). In order to provide evidence for involvement of OCT2 in this process, we next assessed the association of a common germline variant in $S L C 22 A 2$ with changes in serum creatinine in a cohort of cancer patients receiving cisplatin. We focused on the single nucleotide polymorphism (SNP) at the $808 \mathrm{G}>\mathrm{T}$ locus (rs316019), because it is known to be associated with decreased transport of other OCT2 substrates (21), and because of its relatively high allelic frequency (10).

Changes in serum creatinine, a marker of acute nephrotoxicity, were measured at baseline (the day before the first cisplatin administration) and after the first cycle of cisplatin treatment (18 days after the first cisplatin administration) and assessed as a function of $S L C 22 \mathrm{~A} 2808 \mathrm{G}>\mathrm{T}$ genotype. We found that patients carrying a copy of this SNP $(n=10 ; 13 \%)$ experienced no change in serum creatinine after cisplatin treatment $(P=0.12)$, whereas serum creatinine significantly increased in patients carrying the reference sequence $(n=68 ; P=0.0009)$ (Figure 5 ). Differences in serum creatinine levels between baseline and cycle 1 in the patients with the $S L C 22 A 2808 \mathrm{GG}$ genotype remained statistically significant after eliminating the 3 individuals with the largest increase from the analysis $(P=0.00041)$. As predicted based on the results obtained in Oct1/2(-I-) mice, we found that the rs316019 SNP was not associated with the systemic clearance (Figure 6A) or plasma concentrations of cisplatin (Figure 6B). In support of the observed genotype-toxicity association in our patient population, we also found that, in a panel of human cell lines, rs316019 is associated with decreased expression of SLC22A2 in a gene-dosage dependent fashion, although group differences were not statistically significant (data not shown).

\section{DISCUSSION}

In the current study we found that the urinary excretion of cisplatin and drug-induced damage to kidneys is dependent on organic cation transporter-mediated renal tubular transport. This process was found to be mediated by the two closely related murine transporters Oct1 and Oct2 that are functionally redundant, and that together fulfill a role equivalent to that of OCT2 in humans. Furthermore, we noted that individuals carrying an inherited, reduced-function variant in $S L C 22 A 2$, the gene encoding OCT2, are at decreased risk of experiencing cisplatin-induced nephrotoxicity. The current data complement previous knowledge on the interaction of cisplatin with organic cation transporters obtained using in vitro model systems, and may have important practical implications for its optimal use.

As mentioned before, cisplatin has been a mainstay for therapy of multiple solid tumors but its clinical use is hampered by the occurrence of severe and unpredictable side effects to normal tissues, most notably the kidney. Although it has been speculated for several decades that net tubular secretion of cisplatin may be the initiating event in renal tubular damage (6), our demonstration that Oct1/2(-I-) mice exhibit impaired urinary elimination of cisplatin for the 
first time substantiates an important role of organic cation transporters in the in vivo renal handling of this compound. The most plausible explanation for the observed differences in urinary excretion of cisplatin in Oct $1 / 2(-/-)$ mice compared with wildtype mice is an impaired uptake of the drug in renal tubular cells and subsequently reduced renal tubular secretion into urine in the knockout strain. It is noteworthy that compensatory upregulation of various transporter genes occurred in the kidney of Oct $1 / 2(-I-)$ mice, most notably of the Oat 3 gene Slc22a8. However, cisplatin was not found to be transported by either human OAT3 or mouse Oat3, suggesting that in this particular mammalian model, the degree of compensation is not sufficient to counteract the effect of simultaneous deficiency of Oct1 and Oct2.

In spite of the currently observed changes in renal handling of cisplatin in the Oct1/2(-/-) mice, we found that the apparent plasma clearance of cisplatin and measures of systemic exposure to either total cisplatin and unbound cisplatin was unaltered in this mouse model. This paradox might be explained by the possibility that the high expression of Oct1 and Oct2 in renal tubular cells can directly control local drug levels, and thereby alter the urinary excretion of cisplatin without affecting measures of systemic exposure. The notion that the impaired urinary excretion of cisplatin in the Oct1/2(-I-) mice was not accompanied by any evidence of other organ damage indicates a possible shunting of the primary pathway of cisplatin elimination as opposed to a dramatically altered drug distribution. A similar phenomenon has been reported previously for the anticancer and antirheumatic drug methotrexate, where deficiency of Abcc2 (Mrp2), a transporter regulating biliary secretion of this agent, is associated with increases in the extent of urinary excretion compared with wildtype mice (22). Additional investigation is required to confirm the possibility that cisplatin is predominantly eliminated by the hepatobiliary route in the Oct1/2(-/-) mice.

It cannot be entirely excluded that other renal tubular uptake transporters are involved in the urinary excretion of cisplatin. For example, a recent investigation indicated that the copper transporter $1(\mathrm{Ctr} 1 ;$ Slc3lal) is localized on the basolateral side of both proximal and distal tubular cells in the kidney of C57BL/6 mice, and that downregulation of Ctr1 expression by small interfering RNA was associated with decreased cisplatin uptake in vitro (23). In this context, it is interesting to note that the expression of Slc3lal was not different in kidney samples obtained from wildtype mice and Oct1/2(-/-) mice, and was also not affected by treatment with cisplatin (Supp Figure 3). This result indicates a limited involvement for the Ctr1 transporter in the observed pharmacokinetic and pharmacodynamic changes in our particular mouse model.

Recent re-sequencing of the human OCT2 transporter gene SLC22A2 has revealed a number of allelic variants that affect activity of the gene product. Some of these genetic variants may potentially modulate the phenotypic activity of the transporter in patients and therefore affect their predisposition to toxicity and response to substrate drug treatment. In particular, a singlenucleotide polymorphism in exon 4 of the SLC22A2 gene has been described (rs316019), in which a $808 \mathrm{G}>\mathrm{T}$ transversion results in an amino acid change of Serine to Alanine at codon 270 (24). Although a detailed analysis of the potential functional consequences of this $S L C 22 A 2$ variant has not yet been performed, in vitro studies indicated altered substrate specificity and function of the mutant protein relative to the wildtype protein, for example in relation to the OCT2 substrate drug metformin (21). An expression analysis of SLC22A2 in the NCI60 cell line panel suggested that the rs 316019 polymorphism is associated with a decreased gene expression. This change in expression could partially explain the decrease in function observed with this variant, although a more comprehensive evaluation is necessary to draw definite conclusions.

In the present study, we found that white patients with cancer carrying the rs316019 polymorphism $(n=10 ; 13 \%)$ did not experience a significant change in serum creatinine after 
the first cycle of cisplatin treatment, whereas those patients carrying the reference allele showed indications of renal damage. To the best of our knowledge, this is the first evidence suggesting that inherited genetic variation is contributing to interindividual variability in cisplatin-induced nephrotoxicity. Even though this observation requires confirmation in a larger cohort of patients as well as further exploration in other ethnic populations, these findings are in line with those obtained in the Oct1/2(-/-) mice. They also suggest that $a$ priori genetic testing could possibly be employed in order to identify patients, eligible for treatment with cisplatin, that may or may not be at increased risk to develop acute nephrotoxicity.

The current work on the mechanistic basis of cisplatin-induced nephrotoxicity also opens up new avenues for further evaluation of pharmacologic approaches for renoprotection through targeting of OCT2. Particularly, the use of OCT2 inhibitors given combined with cisplatin might prevent high concentrations of cisplatin from reaching the renal tubular cells and subsequently, might reduce the development of nephrotoxicity. Over the last few decades, numerous approaches have been reported to afford renoprotection during cisplatin treatment (25). However, most of these approaches have not separately considered the possible implications on the anticancer actions of cisplatin in tumors, which is surprising considering the overlap and degree of similarity in the critical target genes for cisplatin in the kidney and tumor cell. Indeed, avoiding the occurrence of renal tubular damage following cisplatin treatment by some therapeutic approach is a Phyrric victory if this would be associated with diminished antitumor activity. Our current findings indicate that the expression of OCT2 is very low to absent in solid tumors, in particular in those for which treatment with cisplatin is indicated, such as lung cancer and ovarian cancer, and that OCT2 is unlikely to play a role in the transport of cisplatin into tumor cells in vivo. We are currently investigating whether the administration of an OCT2 inhibitor can ameliorate cisplatin-induced nephrotoxicity in patients with cancer without an influence on circulating cisplatin concentrations.

Collectively, our demonstration that OCT2 plays an important role in cisplatin-related nephrotoxicity reveals a new host factor that contributes to enhanced interindividual variation in tolerability to this drug. These findings might benefit future therapeutic interventions for cisplatin-containing regimens involving the use of specific inhibitors of OCT2.

\section{METHODS}

\section{Cellular Transport}

Human embryonic kidney 293 (HEK293) cells overexpressing human OCT2 were produced using the Flp-In transfection system (Invitrogen, Carlsbad, CA) as previously described (10). Mouse Oat3 cDNA was transfected into HEK293 cells. HEK293 cells overexpressing human OAT3 were provided by Dr. Yuichi Sugiyama (Tokyo, Japan). Measurements of cellular accumulation of cisplatin were performed in six well plates with monolayer cultures at $37^{\circ} \mathrm{C}$ as previously reported (10).

\section{Animal Experiments}

Adult (8-12 week old) male Oct1(-/-), Oct2(-I-) or Oct1/2(-/-) (Taconic, Germantown, NY) mice were used along with age-matched male FVB wildtype mice. Mouse genotypes from tail biopsies were determined using real time PCR with specific probes designed for each gene (Transnetyx, Cordova, TN). A previous investigation has demonstrated that the glomerular filtration rate, assessed using inulin clearance, is not significantly different between Oct1/2(-) mice and FVB wildtype mice (15). All animals were housed and handled in accordance with the Institutional Animal Care and Use Committee of St. Jude Children's Research Hospital. Animals were housed in a temperature controlled environment with a 12 hour light cycle and were given a standard diet and water ad libitum. 
Urinary platinum excretion of cisplatin was studied in animals after they had acclimated to metabolic cages 5 days prior to drug administration. Baseline urine samples were collected sixteen hours after the onset of light, and next, mice were given a single intraperitoneal (i.p.) injection of cisplatin at a dose $10 \mathrm{mg} / \mathrm{kg}$. Urine was collected 4, 24, 48, and 72 hours after administration, diluted with nitric acid $(0.2 \%)$ and immediately analyzed for total platinum using flameless atomic absorption spectrometry (AAS) (10). At the end of the study period, mice were euthanized and tissues harvested for gene expression and histological examination. In separate animals, the plasma pharmacokinetics of cisplatin $(10 \mathrm{mg} / \mathrm{kg}$, i.p. $)$ was determined using a terminal sampling procedure.

\section{Gene Expression Analysis of Mouse Kidney}

RNA was extracted using the RNEasy mini kit (Qiagen, Valencia, CA). RNA samples were amplified from 3 animals per group and then analyzed using the Mouse 430v2 GeneChip array (Affymetrix, Santa Clara, CA). Volcano plots were constructed using select genes, including those that encode enzymes ( $n=27$ probe sets), nuclear receptors $(n=27)$, ABC transporters $(n=56)$ and solute carriers $(n=293)$.

\section{Peripheral Blood Analysis}

Chemistry analysis was performed on serum using a Vetscan autoanalyzer (Abaxis) analyzer to determine levels of albumin, alkaline phosphatase, alanine aminotransferase, amylase, total bilirubin, blood urea nitrogen, calcium, phosphorus, creatinine, glucose, sodium, potassium, and total protein.

\section{Histological Evaluation of Mouse Tissues}

After collection, tissues were fixed overnight in 10\% neutral-buffered formalin. Next, the tissues were processed routinely, embedded in paraffin, sectioned $(4 \mu \mathrm{m})$ and stained with hematoxylin and eosin. Microscopic evaluation was performed by an experienced veterinary pathologist blinded to the composition of the groups. Toxicities, including acute renal tubular necrosis, were scored as 'absent', 'rare', 'mild', 'moderate' or 'severe'.

\section{Real Time PCR and Identification of SLC22A2 Variants in Human Cell Lines}

RNA and DNA from the NCI anti-cancer screening panel were provided by the National Cancer Institute tumor repository (Bethesda, MD). RNA was reverse transcribed using SuperScript III first strand synthesis supermix for qRT-PCR (Invitrogen) according to manufacturer's recommendations. Tissue plates containing cDNA from 48 human tissues and 96 human cancer tissues were obtained from Origene (Rockville, MD). Gene transcripts were quantified using SYBR Green PCR mastermix (Qiagen) and primers previously described (26). Reactions were carried out in triplicate unless otherwise stated as previously reported (10).

The genomic sequence of $S L C 22 A 2$ was obtained by Genbank (accession no. NM 003058) and primers were designed as previously described (10). A single nucleotide polymorphism (SNP) associated with an amino acid change at position Ala270Ser (exon 4) was evaluated. Optimal reaction and cycle conditions were determined for each amplicon in a $25 \mu \mathrm{L}$ reaction volume. As previously described (10) PCR samples were cleaned with ExoSAP-IT reagent (USB Corporation, Cleveland, $\mathrm{OH}$ ) following PCR and sequenced in both forward and reverse directions using Big Dye Terminator (version 3.1) Chemistry on Applied Biosystems 3730XL DNA analyzers. Sequencher software version 4.7 (Gene Codes Corporation, Ann Arbor, MI) was used for sequencing analysis. 


\section{Clinical Studies}

Eligibility criteria, study protocols, and pharmacokinetic analysis for determination of plasma clearance and urinary excretion have been documented in detail previously (27). All patients were treated at the Erasmus MC - Daniel den Hoed Cancer Center (Rotterdam, the Netherlands), the study protocols were reviewed and approved by the Erasmus MC review board, and patients provided written informed consent.

Acute cisplatin-related nephrotoxicity was assessed on the basis on serum creatinine analysis. These measurements were performed on serum samples obtained immediately prior to the first administration of cisplatin (baseline) and on the first serum samples obtained for diagnostic purposes after the first administration of cisplatin. The median time at which the latter samples were obtained was 1 day after start of the cisplatin infusion (range, 1-8 days). Changes in serum creatinine following the administration of cisplatin were expressed as a percentage relative to the baseline values.

\section{Statistical Calculations}

All graphed data is presented as mean values (symbols or bars) with standard error (error bars). Group differences in median pharmacokinetic parameters of cisplatin as a function of mouse genotype or human OCT2-variant status and differences in intracellular drug concentrations in the various cell types were evaluated using a Mann-Whitney $U$ test. Variability in serum chemistry values in the studied mouse strains as a function of treatment was assessed using a Kruskal-Wallis test followed by a Kruskal-Wallis Z-test. Changes in serum creatinine before and after cisplatin treatment in patients was assessed as a function of OCT2-variant status using a Wilcoxon's signed ranks test. Two-tailed $P$ values of less than 0.05 were considered as statistically significant. All statistical calculations were performed using the software package NCSS version 2004 (Number Cruncher Statistical System, Kaysville, UT).

\section{Supplementary Material}

Refer to Web version on PubMed Central for supplementary material.

\section{ACKNOWLEDGMENTS}

We thank Kelli Boyd, David Finkelstein, and John Killmar for invaluable assistance with experiments and data analysis, Yuichi Sugiyama for providing cells overexpressing OAT3, the National Cancer Institute Tumor Repository for providing RNA and DNA from the NCI60 cell lines, and Sharyn Baker and Walter Loos for helpful advice and discussions. This work was supported in part by the American Lebanese Syrian Associated Charities and by United States Public Health Service Cancer Center Support Grant (3P30CA021765).

\section{REFERENCES}

1. Go RS, Adjei AA. Review of the comparative pharmacology and clinical activity of cisplatin and carboplatin. J Clin Oncol 1999;17:409-422. [PubMed: 10458260]

2. Planting AS, van der Burg ME, de Boer-Dennert M, Stoter G, Verweij J. Phase I/II study of a short course of weekly cisplatin in patients with advanced solid tumours. Br J Cancer 1993;68:789-792. [PubMed: 8398709]

3. Arany I, Safirstein RL. Cisplatin nephrotoxicity. Semin Nephrol 2003;23:460-464. [PubMed: 13680535]

4. Berns JS, Ford PA. Renal toxicities of antineoplastic drugs and bone marrow transplantation. Semin Nephrol 1997;17:54-66. [PubMed: 9000550]

5. Yao X, Panichpisal K, Kurtzman N, Nugent K. Cisplatin nephrotoxicity: a review. Am J Med Sci 2007;334:115-124. [PubMed: 17700201]

6. Caterson R, Etheredge S, Snitch P, Duggin G. Mechanisms of renal excretion of cisdichlorodiamine platinum. Res Commun Chem Pathol Pharmacol 1983;41:255-264. [PubMed: 6685336] 
7. Ludwig T, Riethmuller C, Gekle M, Schwerdt G, Oberleithner H. Nephrotoxicity of platinum complexes is related to basolateral organic cation transport. Kidney Int 2004;66:196-202. [PubMed: 15200426]

8. Ciarimboli G, et al. Cisplatin nephrotoxicity is critically mediated via the human organic cation transporter 2. Am.J.Pathol 2005;167:1477-1484. [PubMed: 16314463]

9. Zhang S, et al. Organic Cation Transporters Are Determinants of Oxaliplatin Cytotoxicity. Cancer Res 2006;66:8847-8857. [PubMed: 16951202]

10. Filipski KK, Loos WJ, Verweij J, Sparreboom A. Interaction of cisplatin with the human organic cation transporter 2. Clin Cancer Res 2008;14:3875-3880. [PubMed: 18559608]

11. Vanwert AL, Bailey RM, Sweet DH. Organic anion transporter 3 (Oat3/Slc22a8) knockout mice exhibit altered clearance and distribution of penicillin G. Am J Physiol Renal Physiol 2007;293:F1332-F1341. [PubMed: 17686950]

12. Burger $\mathrm{H}$, et al. Interaction of platinum compounds with the human organic cation transporter hOCT2 (SLC22A2). AACR Meeting Abstracts 2008:698.

13. Litterst CL, LeRoy AF, Guarino AM. Disposition and distribution of platinum following parenteral administration of cis-dichlorodiammineplatinum(II) to animals. Cancer Treat Rep 1979;63:14851492. [PubMed: 498147]

14. Jonker JW, et al. Reduced hepatic uptake and intestinal excretion of organic cations in mice with a targeted disruption of the organic cation transporter 1 (Oct1 [Slc22a1]) gene. Mol Cell Biol 2001;21:5471-5477. [PubMed: 11463829]

15. Jonker JW, Wagenaar E, Van ES, Schinkel AH. Deficiency in the organic cation transporters 1 and 2 (Oct1/Oct2 [Slc22a1/Slc22a2]) in mice abolishes renal secretion of organic cations. Mol.Cell Biol 2003;23:7902-7908. [PubMed: 14560032]

16. Koepsell H, Lips K, Volk C. Polyspecific organic cation transporters: structure, function, physiological roles, and biopharmaceutical implications. Pharm Res 2007;24:1227-1251. [PubMed: 17473959]

17. Sweet DH, Miller DS, Pritchard JB, Fujiwara Y, Beier DR, Nigam SK. Impaired organic anion transport in kidney and choroid plexus of organic anion transporter 3 (Oat3 (Slc22a8)) knockout mice. J Biol Chem 2002;277:26934-26943. [PubMed: 12011098]

18. de Jongh FE, et al. Body-surface area-based dosing does not increase accuracy of predicting cisplatin exposure. J Clin Oncol 2001;19:3733-3739. [PubMed: 11533095]

19. Safirstein R, Winston J, Moel D, Dikman S, Guttenplan J. Cisplatin nephrotoxicity: insights into mechanism. Int J Androl 1987;10:325-346. [PubMed: 3583421]

20. Stewart DJ, Dulberg CS, Mikhael NZ, Redmond MD, Montpetit VA, Goel R. Association of cisplatin nephrotoxicity with patient characteristics and cisplatin administration methods. Cancer Chemother Pharmacol 1997;40:293-308. [PubMed: 9225947]

21. Song IS, et al. Genetic variants of the organic cation transporter 2 influence the disposition of metformin. Clin Pharmacol Ther 2008;84:559-562. [PubMed: 18401339]

22. Vlaming ML, et al. Impact of Abcc2 (Mrp2) and Abcc3 (Mrp3) on the in vivo elimination of methotrexate and its main toxic metabolite 7-hydroxymethotrexate. Clin Cancer Res 2008;14:81528160. [PubMed: 19088030]

23. Pabla N, Murphy RF, Liu K, Dong Z. The copper transporter Ctr1 contributes to cisplatin uptake by renal tubular cells during cisplatin nephrotoxicity. Am J Physiol Renal Physiol 2009;296:F505-F511. [PubMed: 19144690]

24. Fujita T, Urban TJ, Leabman MK, Fujita K, Giacomini KM. Transport of drugs in the kidney by the human organic cation transporter, OCT2 and its genetic variants. J Pharm Sci 2006;95:25-36. [PubMed: 16307453]

25. Pabla N, Dong Z. Cisplatin nephrotoxicity: mechanisms and renoprotective strategies. Kidney Int 2008;73:994-1007. [PubMed: 18272962]

26. Shu Y, Bello CL, Mangravite LM, Feng B, Giacomini KM. Functional characteristics and steroid hormone-mediated regulation of an organic cation transporter in Madin-Darby canine kidney cells. J Pharmacol Exp Ther 2001;299:392-398. [PubMed: 11561104]

27. Loos WJ, et al. Evaluation of an alternate dosing strategy for cisplatin in patients with extreme body surface area values. J Clin Oncol 2006;24:1499-1506. [PubMed: 16574999] 

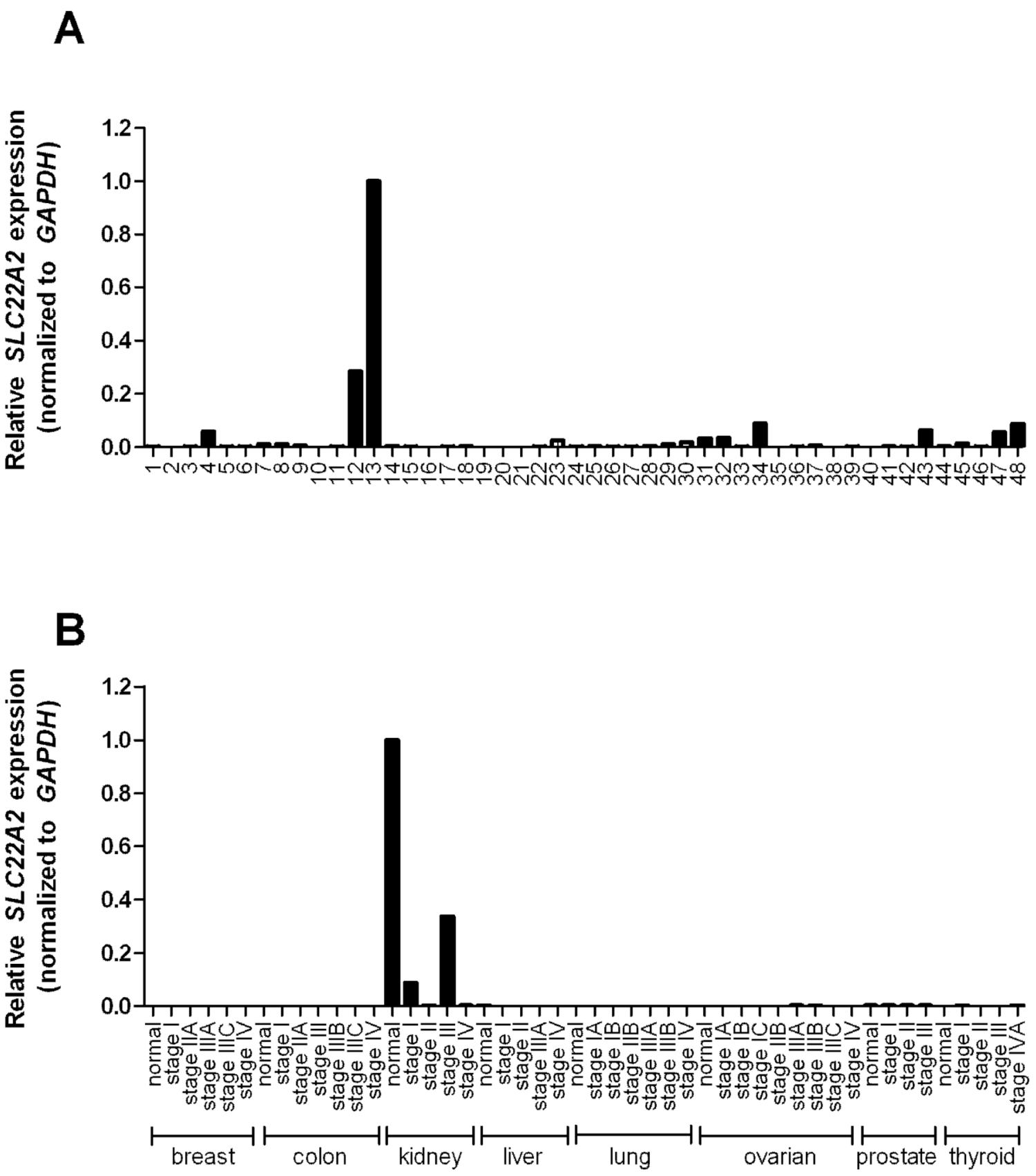

Figure 1.

Expression of $S L C 22 A 2$, normalized to the house keeping gene $G A P D H$, in (A) human normal tissues and (B) human tumor samples. SLC22A2 was predominantly expressed in kidney and low to absent in tumor samples. Tissue and tumor plates containing cDNA tissues were used for real-time PCR analysis. Data are shown as mean values (symbols) of duplicate plates and expressed relative to values observed in normal human kidney, which was set to a value of 1 . Numbers represent tissue from (1) Adrenal gland, (2) Bone marrow, (3) Brain, (4) Cervix, (5) Colon, (6) Descending duodenum, (7) Epididymis, (8) Esophagus, (9) Fat, (10) Heart, (11) Small intestine, (12) Intracranial artery, (13) Kidney, (14) Liver, (15) Lung, (16) Lymph node, (17) Plasma blood leucocyte, (18) Mammary gland, (19) Muscle, (20) Nasal mucosa, (21) 
Optic nerve, (22) Ovary, (23) Oviduct, (24) Pancreas, (25) Penis, (26) Pericardium, (27) Pituitary, (28) Placenta, (29) Prostate, (30) Rectum, (31) Retina, (32) Seminal vesicles, (33) Skin, (34) Spinal cord, (35) Spleen, (36) Stomach, (37) Testis, (38) Thymus, (39) Thyroid, (40) Tongue, (41) Tonsil, (42) Trachea, (43) Urethra, (44) Urinary bladder, (45) Uterus, (46) Uvula, (47) Vagina, (48) Vena cava. 


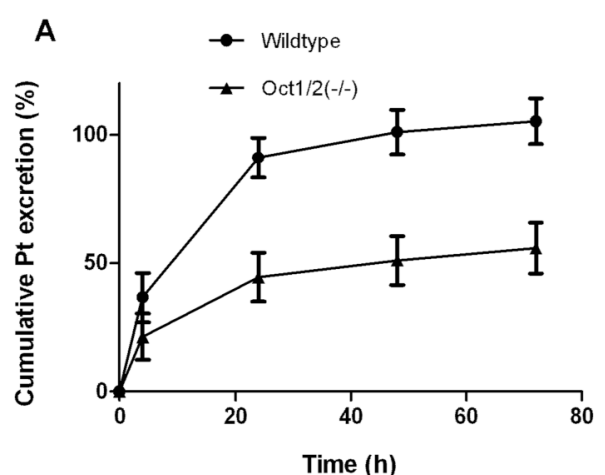

C

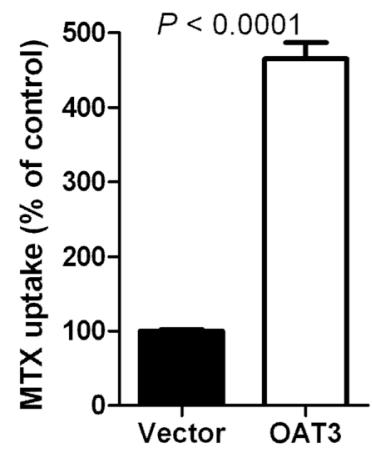

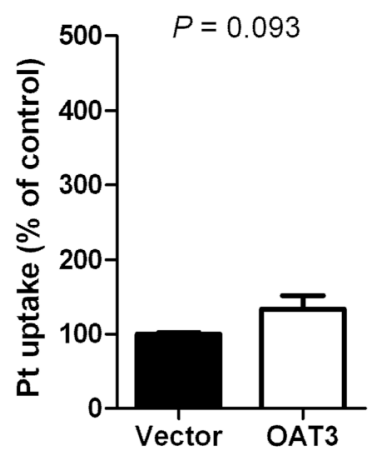

B
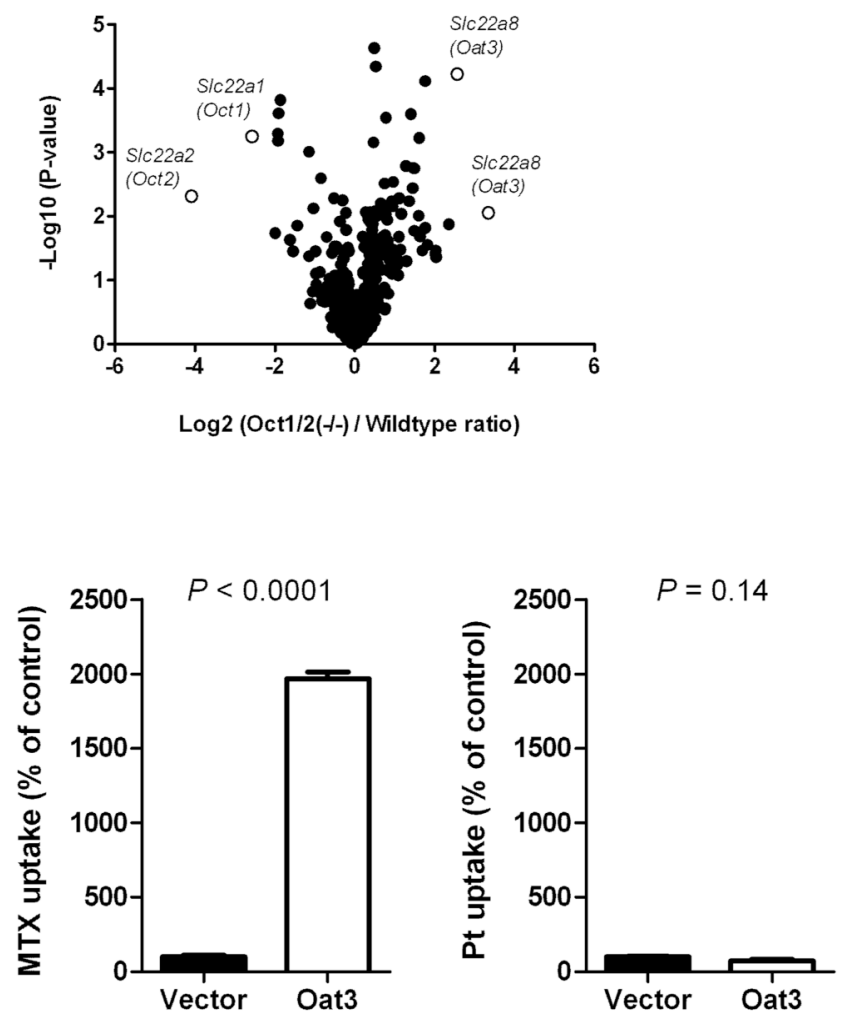

Figure 2.

Urinary platinum excretion and gene expression changes in $\operatorname{Oct1}(-/-)$ and $\operatorname{Oct} 2(-/-)$ mice.

(A) Effect of simultaneous Oct1 and Oct2 deficiency on renal handling of cisplatin in mice. The cumulative excretion of cisplatin was reduced in Oct1/2(-/-) mice compared to wildtype mice ( $n=11-12 /$ group) after drug administration $(10 \mathrm{mg} / \mathrm{kg}$; i.p.). Data are shown as mean values; error bars represent standard error. (B) Differential gene expression in the kidney of male Oct1/2(-/-) mice relative to wildtype FVB mice ( $n=3 /$ group) assessed using the Affymetrix Mouse 430v2 GeneChip array. Select genes on the volcano plot include enzymes, nuclear receptors, ABC transporters, and solute carriers. (C) The intracellular uptake of methotrexate (MTX), a positive control, and platinum (Pt) was assessed in HEK293 cells transfected with human OAT3 and mouse Oat 3 following incubation of $10 \mu \mathrm{M}$ or $500 \mu \mathrm{M}$ methotrexate or $500 \mu \mathrm{M}$ cisplatin for a period of 30 minutes. Data are expressed relative to drug accumulation in cells transfected with an empty vector, which was set to $100 \%$. Data are shown as mean values with standard error. 
A

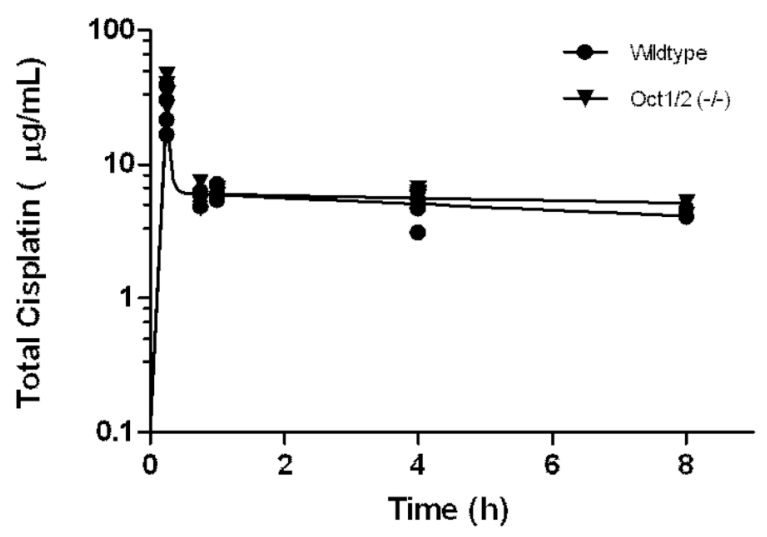

B

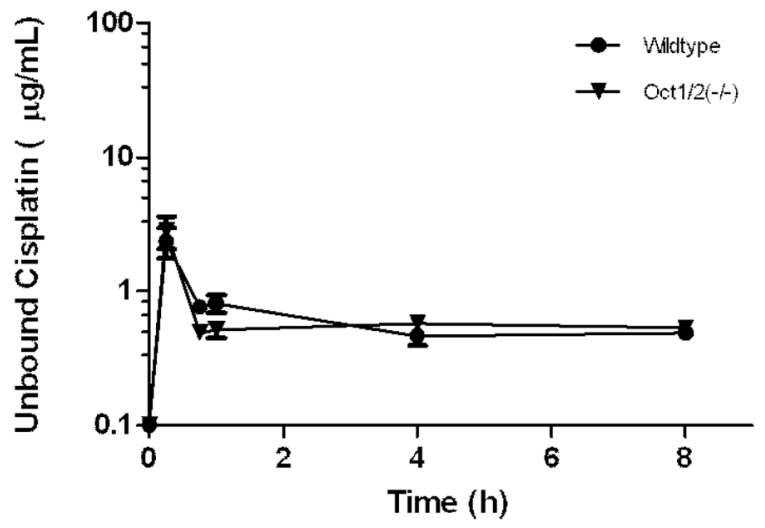

Figure 3.

Plasma pharmacokinetics in mice after cisplatin treatment. (A) Comparative concentrationtime profiles of cisplatin in plasma of $\operatorname{Oct} 1 / 2(-/-)$ mice $(\boldsymbol{\nabla})$ and wildtype mice $(\bullet)(n=4 /$ group) after the administration of cisplatin (10 mg/kg; i.p.). Data are shown as mean values with standard error along with a curve fit from a 2 compartment model. (B) Comparative concentration-time profiles of unbound platinum in plasma of $\operatorname{Oct} 1 / 2(-/-)$ mice $(\boldsymbol{\nabla})$ and wildtype mice $(\bullet)$ ( $n=4 /$ group) after the administration of cisplatin $(10 \mathrm{mg} / \mathrm{kg}$, i.p.). Data are shown as mean values with standard error. 
A

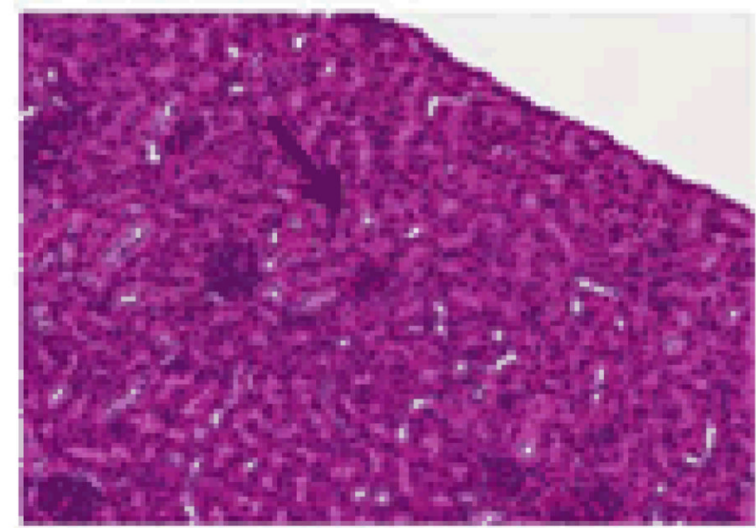

$\mathrm{c}$

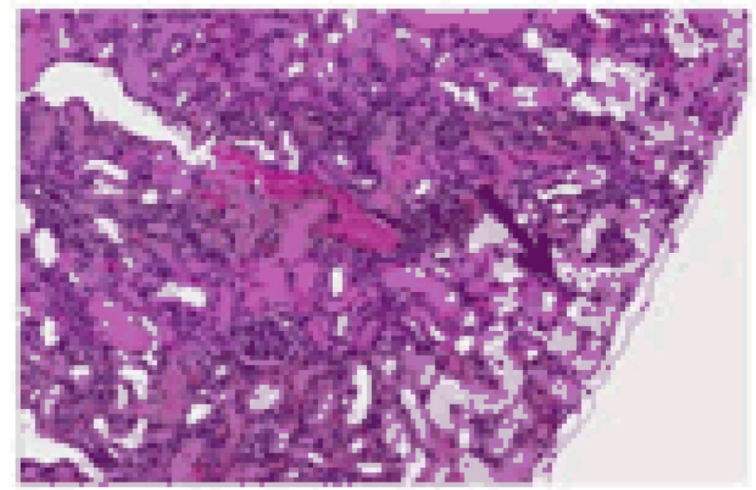

B.

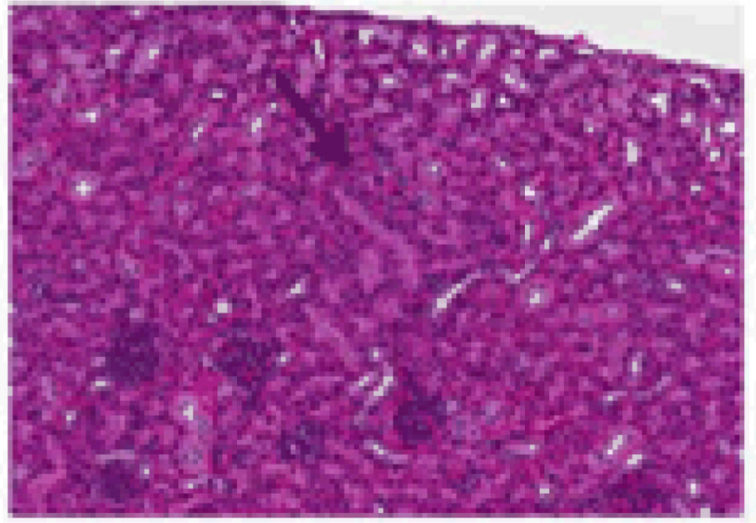

D

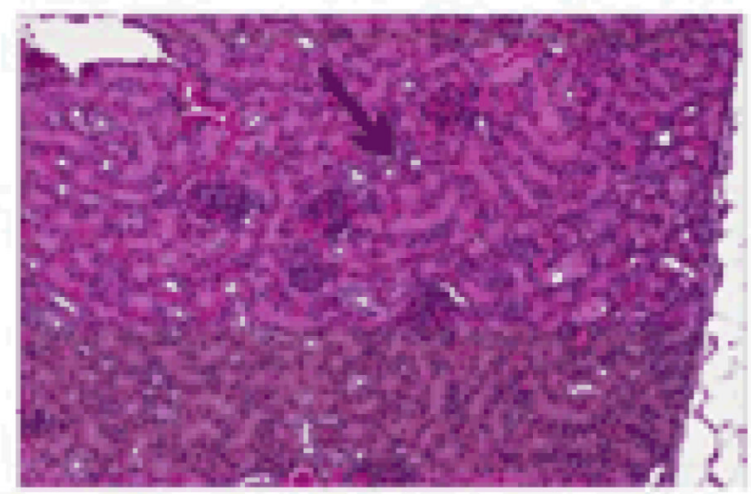

Figure 4.

Comparative cisplatin-related nephrotoxicity in (A) untreated wildtype and (B) untreated Oct1/2(-/-) mice with $(\mathbf{C})$ treated wildtype, and (D) treated Oct1/2(-/-) mice 72 hours after the administration of cisplatin $(10 \mathrm{mg} / \mathrm{kg}$, i.p.) from representative animals. Severe renal tubular necrosis, characterized by dilated tubules filled with necrotic tubular epithelial cells, was observed in kidneys of all wildtype mice but in none of the Oct1/2(-/-) mice ( $n=8 /$ group). Arrows indicate tubules. 


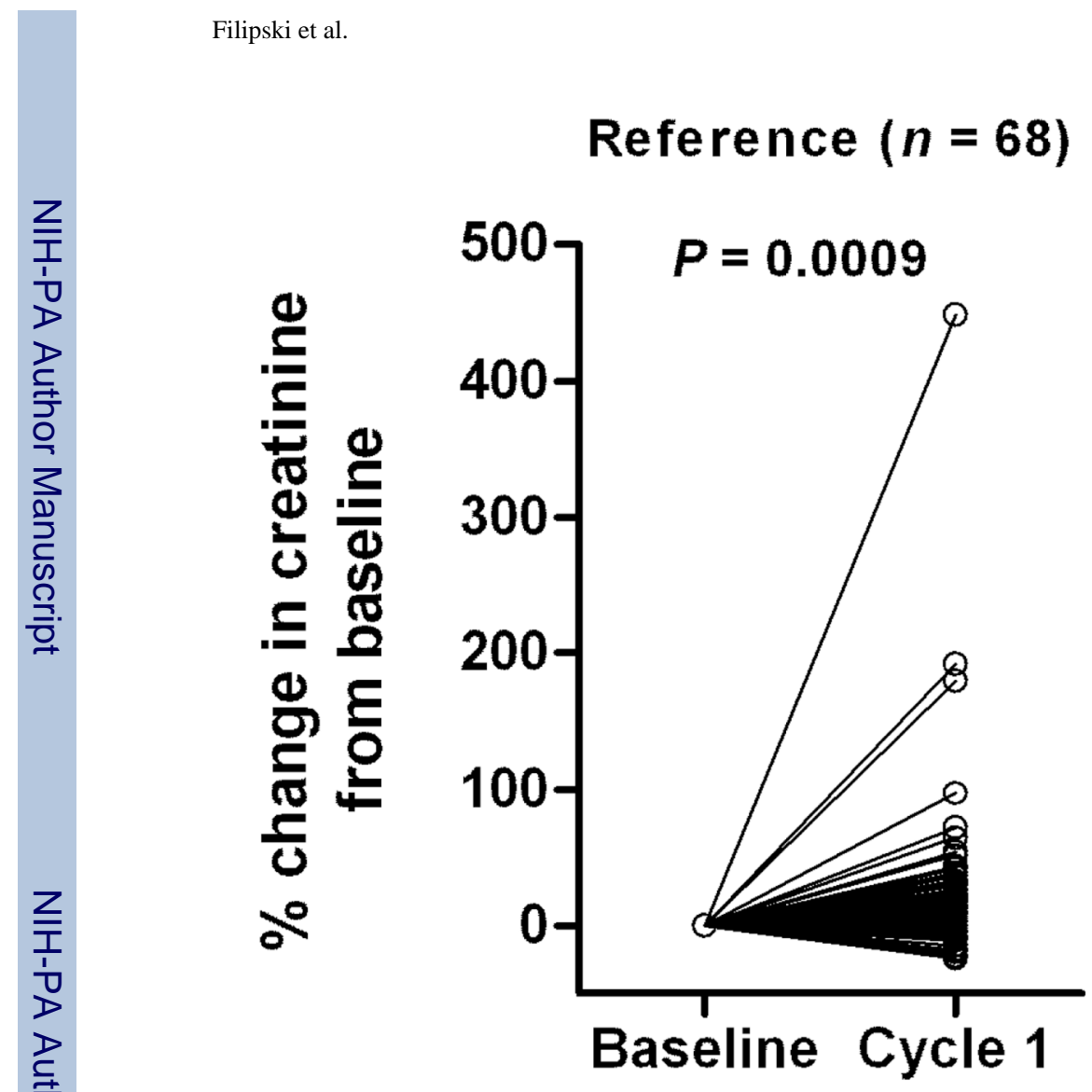

Variant $(n=12)$

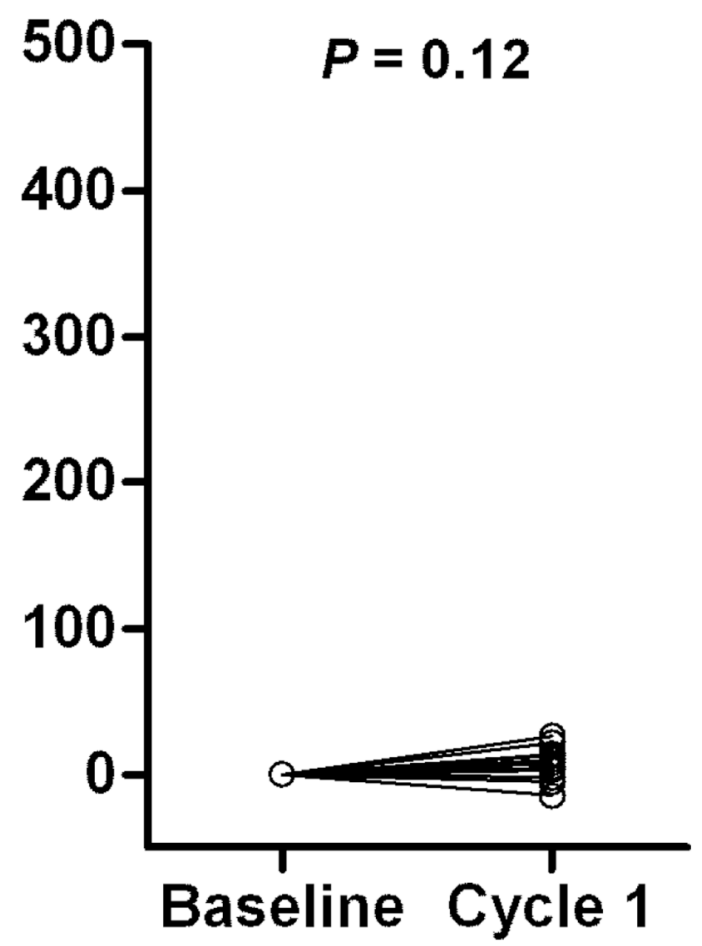

Figure 5.

Changes in serum creatinine, a marker of acute nephrotoxicity, measured at baseline and after the first cycle of cisplatin treatment in cancer patients as a function of SLC22A2 808G>T genotype (GG; $n=68, \mathrm{GT} ; n=10)$. 
A

$$
P=0.69
$$

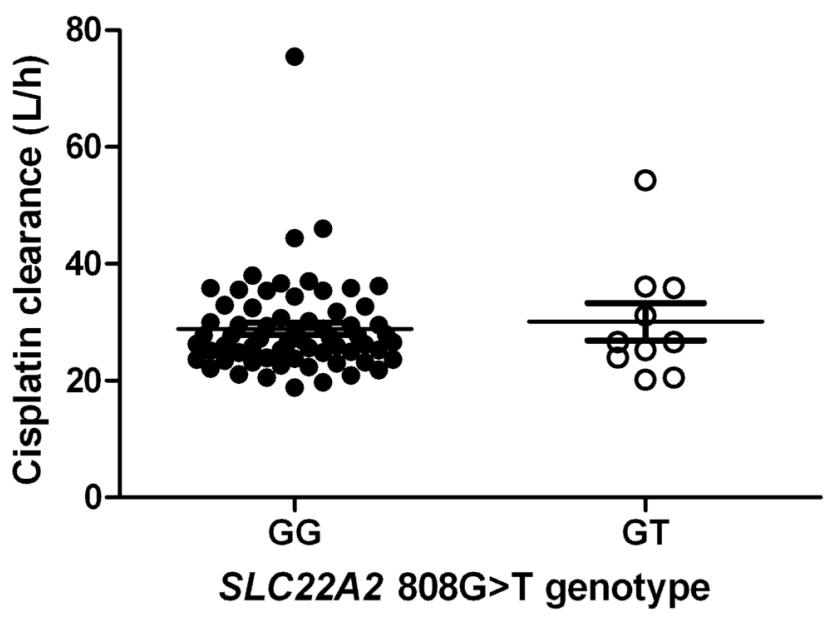

B

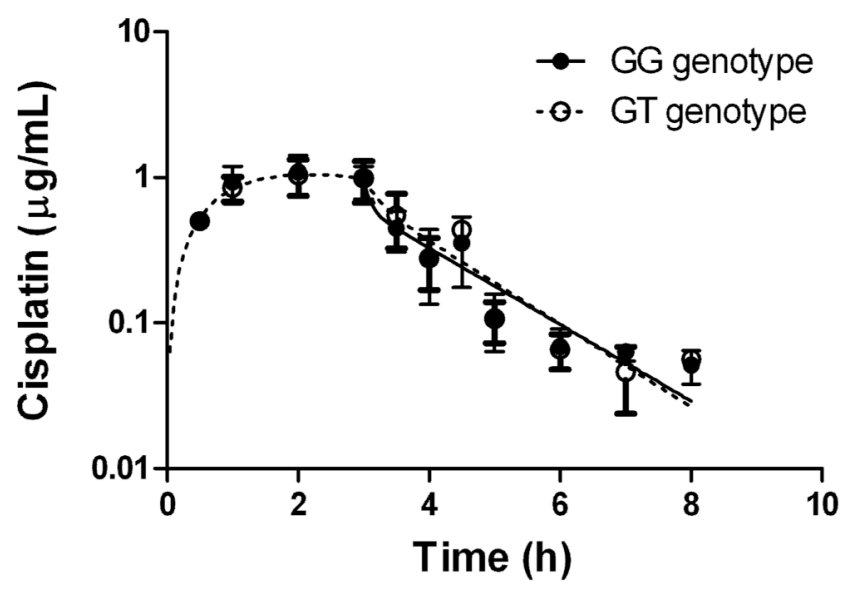

Figure 6.

(A) Unchanged systemic clearance of unbound cisplatin in cancer patients as a function of SLC22A2 808G>T genotype status (GG; $n=68$, GT; $n=10$ ). Each point represents a patient and mean values are indicated by lines. (B) Concentration-time profile of unbound cisplatin as a function of $S L C 22 A 2808 \mathrm{G}>\mathrm{T}$ genotype status (GG; $n=68, \mathrm{GT} ; n=10$ ). Data are shown as mean values \pm standard error along with a curve fit from a 2 -compartment model (lines). 


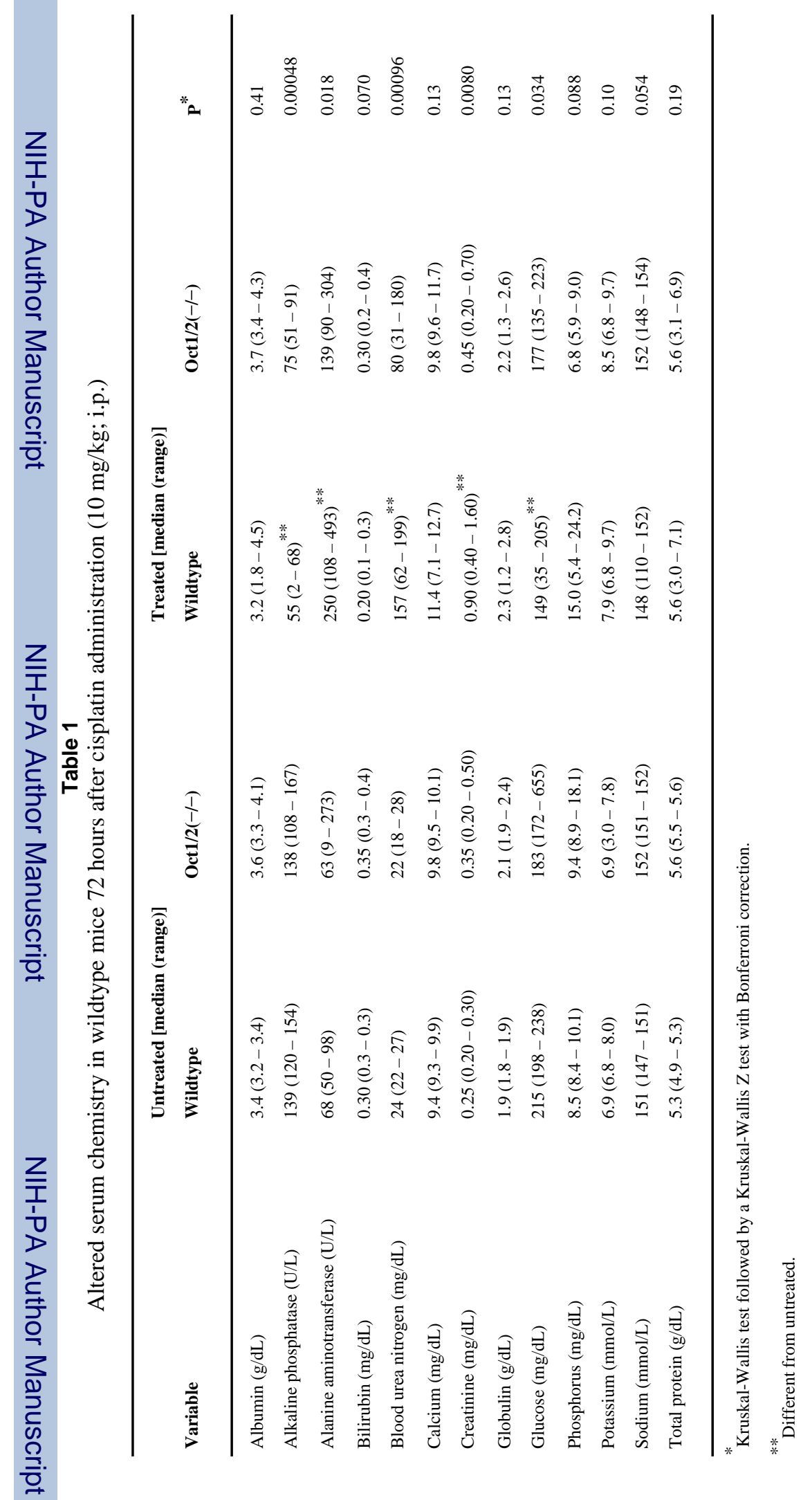

